\title{
Is it possible to tactually recognize dural puncture resistance during puncture for spinal anesthesia?
}

\author{
Isao Utsumi, Sachiko Omi, Tomasz Hascilowicz \\ The Jikei University School of Medicine
}

\section{INTRODUCTION}

While performing spinal subarachnoid anaesthesia, puncture of the dura / arachnoid is difficult to confirm directly and is only noticeable through a subjective tactual sensation. This is why the cerebrospinal fluid reflux serves as the main indicator of the correct needle placement. In some cases, however, the tactile sensation during puncture of the dura mater (referred to as "pop" or "dural click") indicates the moment when the needle progression should be stopped even before CSF reflux is confirmed. The "pop" sensation itself, however, has not been considered a sufficient indicator of the dural / arachnoid puncture. It is a subjective sensation generally recognised by anaesthetists, but it has not been studied before. We investigated whether it is possible to tactually recognize the moment of puncture caused by dural and arachnoid resistance during puncture performed with spinal anaesthesia needles and how accurate is this sensation.

Figure. 1 Load deformation curves

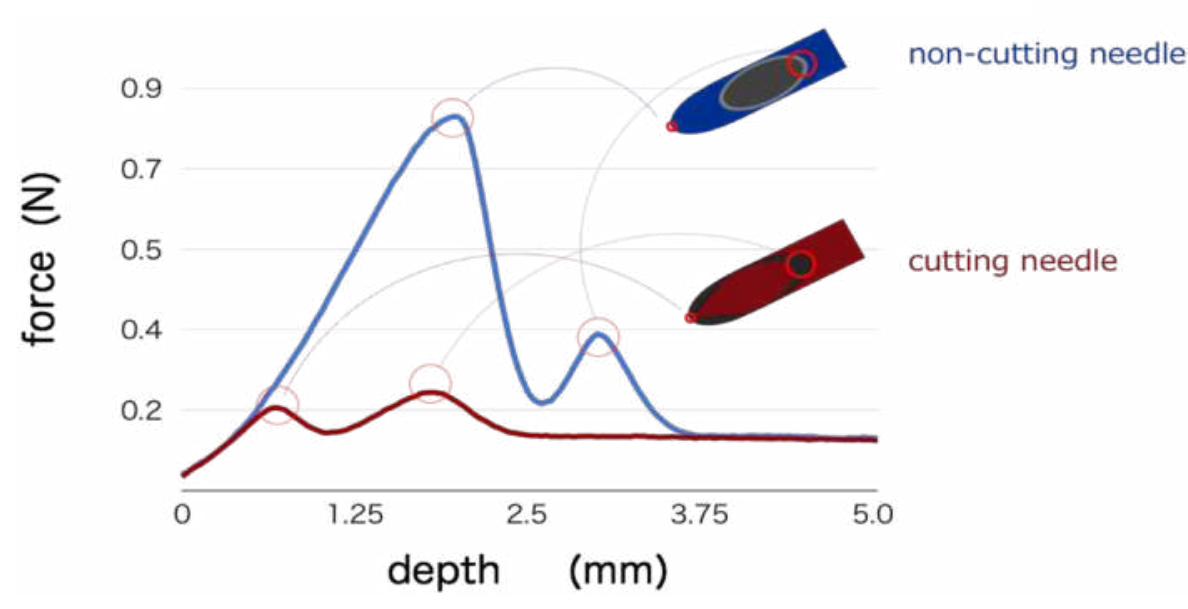

\section{METHOD}

Fifty physicians experienced in spinal anaesthesia procedure were recruited.

Seven needles were chosen so that the comparison would not be complicated. (Table 1) All needle hubs were covered with tapes of different colours so that needle shapes and gauges could not be distinguished from the needle appearance.

PA (polyamide: nylon) film was used as substitute of dura mater.

(Thickness of duras: human: 250 to $400 \mu \mathrm{m}$, porcine: 125 to $485 \mu \mathrm{m}$, PA film: $50 \mu \mathrm{m}$ )

Puncture resistance produced by each spinal needle was recorded in advance as "load deformation curve" (Figure 1) using a universal mechanical testing machine (AG-I: Shimadzu Corporation).

Model: Each spinal needle was set through a fixed $20 \mathrm{G}$ guide needle with its tip $1 \mathrm{~cm}$ just above the artificial dura and the punctures were easily performed only by pushing the needle in vertical direction.

Comparison method: First, all participants punctured the artificial dura with the 25G Dr.Japan's "facil point" needle and the tactile sensation felt upon puncture with this needle was used as referrence and set to "10 points" on a numeric rating scale (NRS). Next, all participants punctured the artificial dura with 7 kinds of different spinal needles and the strength of puncture resistance subjectively felt by each participant's fingertips was evaluated with NRS.

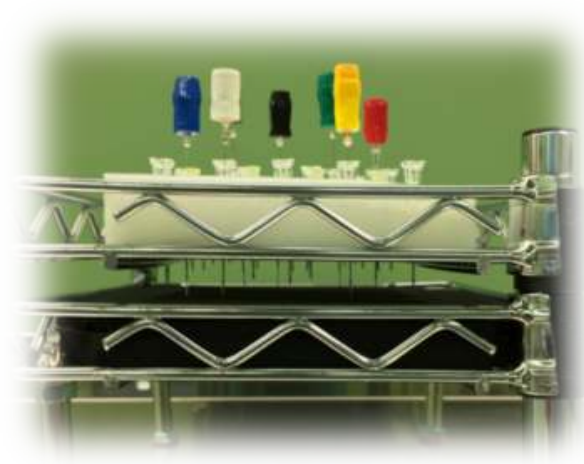

\section{RESULTS AND DISCUSSION}

All participants could discriminate differences in puncture resistance produced by different spinal needles and the average NRS values correlated with the measured puncture resistances (Table 1). Furthermore, the accurate tactile recognition threshold was $0.26 \mathrm{~N}$ or more among $80 \%$ of participants, and $90 \%$ of participants could accurately distinguish differences between needles producing $0.71 \mathrm{~N}$ or higher resistance upon puncture of the artificial dura (Figure 2). The measured puncture resistances produced by pencil point needles were higher than those obtained with cutting needles of the same gauges, and this needle shape differences could be also tactually recognised by $80 \%$ of participants with needles producing $0.26 \mathrm{~N}$ or higher resistances. The high puncture resistance produced by the facil point needle was thought to be due to the large step difference between the stylet and the outer cylinder.

\begin{tabular}{|c|c|c|c|c|c|c|}
\hline \multicolumn{1}{|c|}{ Table. 1} & $\begin{array}{c}\text { needle } \\
\text { shape }\end{array}$ & \multicolumn{3}{c}{ Geak of Resistance } & \multicolumn{2}{c|}{ NRS } \\
& porcine dura & PA film & average & SD \\
\hline UNISIS & cutting & 27 & $0.17 \mathrm{~N}$ & $0.35 \mathrm{~N}$ & 2.48 & 1.8 \\
\hline UNISIS & cutting & 25 & $0.21 \mathrm{~N}$ & $0.39 \mathrm{~N}$ & 3.54 & 1.8 \\
\hline B-Braun & cutting & 25 & $0.39 \mathrm{~N}$ & $0.68 \mathrm{~N}$ & 4.46 & 2.1 \\
\hline UNISIS & 27 & $0.61 \mathrm{~N}$ & $0.81 \mathrm{~N}$ & 4.94 & 1.6 \\
\hline UNISIS & pencil & 25 & $0.66 \mathrm{~N}$ & $0.85 \mathrm{~N}$ & 5.66 & 1.8 \\
\hline B-Braun & pencil & 25 & $0.94 \mathrm{~B}$ & $1.27 \mathrm{~N}$ & 7.22 & 2 \\
\hline Dr.Japan
\end{tabular}

\section{LIMITATION}

$\%$

\% $\quad$ Figure.2

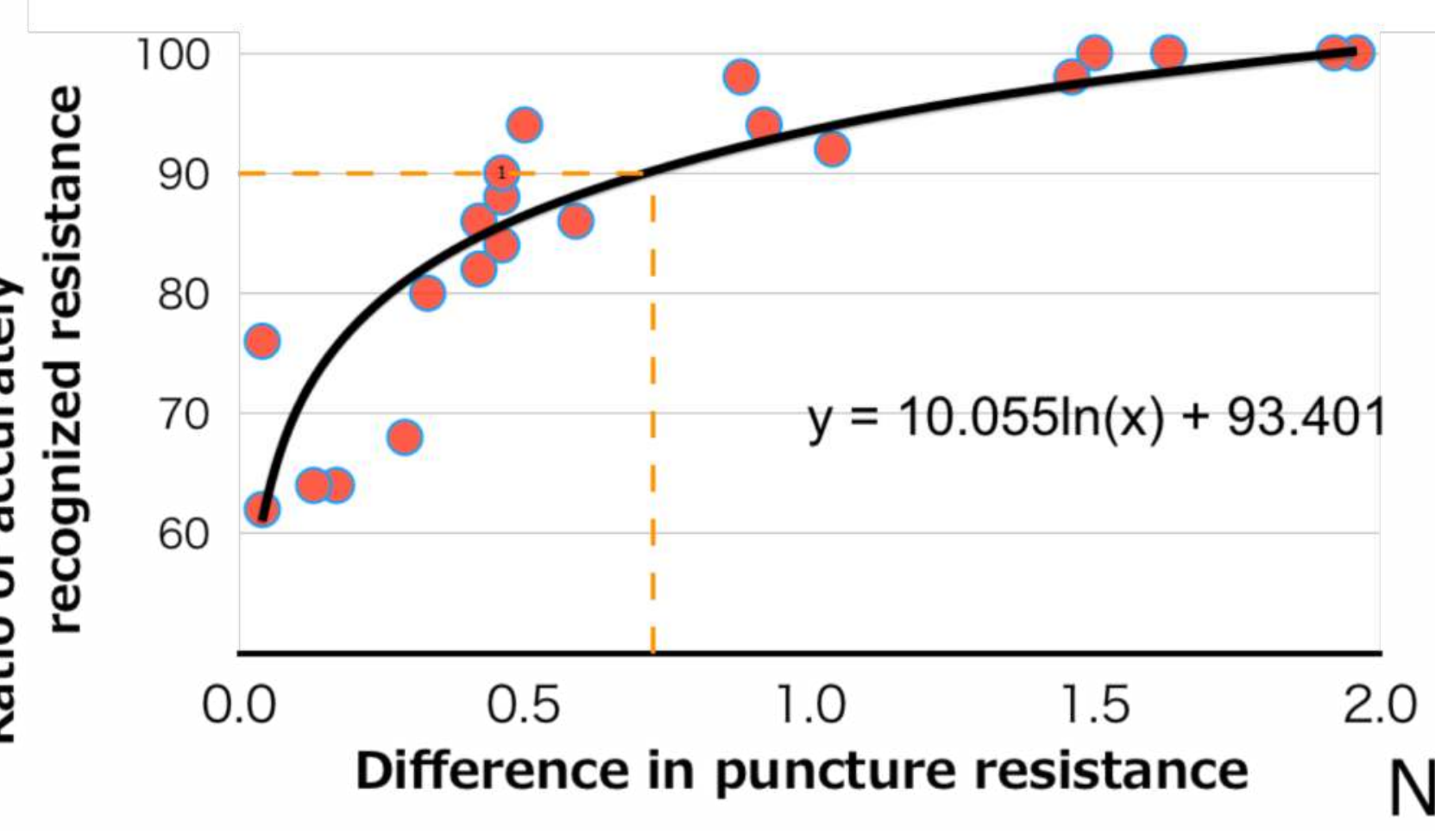

Artificial dura mater (PA film) with load-deformation curve and puncture resistance close to porcine dura was selected. The biological dura mater is a fibrous tissue with structural and thickness differences observed both between individual animals and its different regions. It was not used because of this variety that could possibly affect the results. Furthermore, during an actual spinal puncture, the needle passes other tissues before reaching the dura and additional friction resistances are possibly produced. If a guide needle is used and its tip reaches the epidural space, the resistance obtained by the spinal needle should be almost produced by perforation of the dura mater.

\section{CONCLUSION}

In spinal anaesthesia, higher resistances felt on dural puncture should facilitate more direct and easier understanding of the moment when dura mater is actually punctured. A clearly distinguishable "dural click" would be beneficial since:

1) the number of punctures might be reduced, 2) sensitivity of the procedure might increase, and, 3) the procedure would be easier to train.

We think that selection/manufacture of a spinal needle should involve consideration of the aspects related to needle resistance. Thus, the procedure should become easier and eventually safer. 